\title{
Abstract \\ The Extent of Consequential DNA Damage in Human Tumors from TCGA PanCanAtlas ${ }^{\dagger}$
}

\author{
Aleksey V. Belikov*(D), Alexey D. Vyatkin, Danila V. Otnykov and Sergey V. Leonov \\ Laboratory of Innovative Medicine, School of Biological and Medical Physics, Moscow Institute of Physics and \\ Technology, 141701 Dolgoprudny, Moscow Region, Russia; vyatkin.ad@phystech.edu (A.D.V.); \\ dan-otn@yandex.ru (D.V.O.); leonov.sv@mipt.ru (S.V.L.) \\ * Correspondence: belikov.research@gmail.com \\ † Presented at the 1st International Electronic Conference on Cancers: Exploiting Cancer Vulnerability by \\ Targeting the DNA Damage Response, 1-14 February 2021; Available online: https:/ /iecc2021.sciforum.net/.
}

check for updates

Citation: Belikov, A.V.; Vyatkin, A.D.; Otnykov, D.V.; Leonov, S.V. The Extent of Consequential DNA Damage in Human Tumors from TCGA PanCanAtlas. Med. Sci. Forum 2021, 3, 4. https://doi.org/ 10.3390/IECC2021-09202

Academic Editors: Nicola Curtin and Helen E. Bryant

Published: 29 January 2021

Publisher's Note: MDPI stays neutral with regard to jurisdictional claims in published maps and institutional affiliations.

Copyright: () 2021 by the authors. Licensee MDPI, Basel, Switzerland. This article is an open access article distributed under the terms and conditions of the Creative Commons Attribution (CC BY) license (https:// creativecommons.org/licenses/by/ $4.0 /)$.

\begin{abstract}
DNA damage is crucial for the emergence of cancer cells. If the DNA damage response is defective, the DNA damage is converted to fixed mutations. Some of these mutations drive tumorigenesis and are called driver mutations. However, the extent of consequential DNA damage per tumor, i.e., the number of various kinds of driver mutations, is not known. We utilized the largest database of human cancer mutations (TCGA PanCanAtlas), multiple popular algorithms for cancer driver prediction, and several custom scripts to estimate the number of various kinds of driver mutations in primary tumors. We found that there were on average 22 driver mutations per patient's tumor, of which 1.7 were point mutations in oncogenes, 8.5 were amplifications of oncogenes, 1.6 were point mutations in tumor suppressors, 3.9 were deletions of tumor suppressors, 1.5 were driver chromosome losses, 1 was driver chromosome gain, 2 were driver chromosome arm losses, and 1.5 were driver chromosome arm gains. The number of driver mutations per tumor increased with age, from 11.2 for 25 y.o. to 28.6 for $>85$ y.o. The number of driver mutations per tumor varied strongly between cancer types, from 1.9 in thyroid carcinoma to 49 in lung squamous cell carcinoma. Overall, our results provide valuable insights into the extent of functional DNA damage in tumors.
\end{abstract}

Keywords: cancer; tumor; driver; mutation; gene; arm; chromosome; oncogene; suppressor; amplification; deletion; gain; loss; SNA; CNA; aneuploidy; TCGA; PanCanAtlas; age; gender; cohort

Supplementary Materials: The following are available online at https: / /www.mdpi.com/article/ 10.3390/IECC2021-09202/s1.

Institutional Review Board Statement: Not applicable.

Informed Consent Statement: Not applicable.

Data Availability Statement: Data and code supporting reported results can be found at https: / / github.com/belikov-av (accessed on 1 January 2021). 